\title{
Study On The Remote Monitoring System For Medical Insurance Prescription Of Chinese Patent Medicine
}

\author{
Wangping Xiong ${ }^{1}$, Jianqiang $\mathrm{Du}^{1}$, Bin $\mathrm{Nie}^{1}$, Xian $\mathrm{Zhou}^{1, \mathrm{a}}$ \\ ${ }^{1}$ School of Computer, Jiang Xi University of Traditional Chinese Medicine, NanChang, JiangXi, China
}

\begin{abstract}
Chinese patent medicine was the preparation and integration of traditional Chinese medicine in $t$ raditional Chinese medicine.It was a continuation of traditional Chinese medicine in modern society.China's hospital information construction was at a stage of development.The implementation of the medical insuran ce prescription monitoring system had significantly improved the level of medical insurance service manage ment in hospitals. Based on the Eclipse platform and MYSQL database, this paper constructed a prescriptio $\mathrm{n}$ insurance monitoring system for Chinese patent medicines.A visual graph of drug monitoring and medicat ion recording was formed to determine whether there is any unreasonable phenomenon. At the same time,au xiliary diagnosis needed to input common symptoms of disease into database. According to the search,it cou ld respond quickly,assist doctors to diagnose and improve the speed of seeking medical treatment. Prescripti on management recorded the prescribed prescriptions to achieve the visualization of prescription manageme nt,and achieved maximum maintainability and operability.
\end{abstract}

\section{Introduction}

Chinese patent medicine has the advantages of definite cu rative effect,convenient use and little side effects.Under th e condition of reasonable use, the safety of Chinese patent medicines is relatively high.However,in the course of actu al use,problems such as repeated medication,inappropriate indications, excessive course of treatment and excessive $d$ osage often occur.This requires a great deal of attention.In order to solve this series of problems, it is necessary to mo nitor the information system to avoid some abnormal med ical insurance problems,such as"hedging","fraud"and so o n.

The informatization of medical insurance managemen $t$ is mainly dependent on the development of Internet tech nology, combining with the advanced management mode and concept, replacing the manual management with the computer operation, abandoning the traditional paper file management mode, building a flexible and rapid manage ment system, which is more conducive to the relevant dep artments to insure information management of personnel. The development of information age makes the automati on and intelligence of medical insurance management po ssible, which provides great convenience for the work of medical insurance administrators. The development of inf ormation age makes the automation and intelligence of $\mathrm{m}$ edical insurance management possible, which provides gr eat convenience for the work of medical insurance admin istrators. Informatization of medical insurance manageme nt is a higher requirement,and it is also the best form of " dead files" to become "live files".

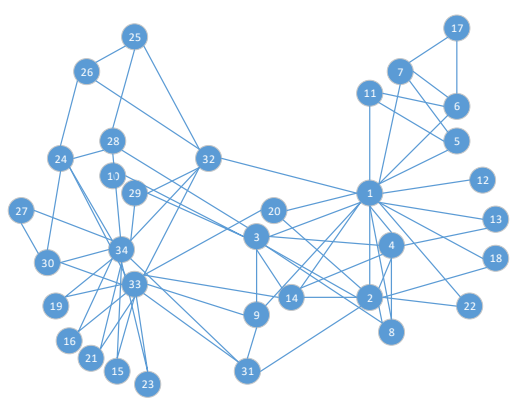

Figure 1. Component-Target network diagram.

With the progress of universal medical insurance,the $t$ otal number of basic medical insurance coverage has gra dually increased.In the face of more and more insured res idents, how to do well the management of medical insuran ce is an important problem facing the medical insurance $\mathrm{i}$ $\mathrm{n}$ today's society.In order to realize the security,efficiency, simplicity and practicality of medical insurance informati on management under the current Internet technology en vironment,From the perspective of science and technolog $y$ development,manual paper files are no longer suitable $f$ or the management of medical insurance.Informatization and automation will be the trend of medical insurance ma nagement in the future. How to adapt the medical insuran ce management to the demands of the residents' insuranc e and the demand of scientific and technological progress, to realize the modernization and information of manage ment is the practical problem that the staff of the medical insurance departments and the developers of the system must be confronted with.Realizing the modernization and informationization of management is a problem that mus 
$\mathrm{t}$ be faced by the staff of the medical insurance departme $\mathrm{nt}$ and the developers of the system development.

With the rapid development of information technolog $y$, the quality of prescription must be improved by means of information technology. The use of medical insurance prescription monitoring system in Chinese patent medici ne will be of great significance:

(1)Interventions for outpatient prescriptions for irratio nal Chinese patent medicines to standardize prescribing $b$ ehavior.

Regularly check the prescriptions issued by doctors a nd check the quality of prescription.After the operation of the medical insurance prescription monitoring system,th e electronic prescription information is classified and pro cessed.At the same time,some unreasonable"big Prescript ions"and other phenomena in the outpatient department were intervened in real time, and the prescription behavio $r$ was further standardized and the medical insurance pro blem was eliminated to the maximum.

(2)Improve the ability of rational use of medical reso urces.

At present, the national medical insurance expenditure mainly comes from the payment of units and individuals, as well as the transfer payment by the government.Due to the excessive use of medical insurance resources and oth er reasons,there was a huge gap in the annual medical ins urance expenditure in the whole country. The use of the $\mathrm{m}$ edical insurance prescription monitoring system can mak e the hospital administrators allocate the medical resourc es reasonably,master all kinds of information in real time, and provide the basis for management and control and de cision-making.

With the continuous improvement of medical insuran ce coverage,the number of medical insurance prescription $\mathrm{s}$ in each hospital has increased rapidly.At the same time, the demand for medical insurance service monitoring abil ity is getting higher and higher in China.Some system sof tware in this area is still in a stage of development,and it $i$ s not very clear.But now environmental demand is strong, monitoring system is the general trend.

The construction and implementation of the medical $i$ nsurance service monitoring system will help the hospital to improve the breadth and depth of the medical insuranc e monitoring and management,and help the rational distri bution of medical resources in the hospital,and help the $p$ atients to use medical insurance resources reasonably. The construction of hospital informatization in China is at a $\mathrm{s}$ tage of development. The implementation of the medical insurance prescription monitoring system will significantl $\mathrm{y}$ improve the level of medical insurance service manage ment in hospitals.Because the construction of digital hosp ital is constantly improving, the function of medical insur ance service monitoring will continue to develop along $\mathrm{w}$ ith the construction of hospital informatization. Only in th e process of construction can we continuously explore an d sum up experience to make transparent medical insuran ce policies serve the society better.

\section{Comprehensive requirements of the system}

\subsection{Reliability and availability requirements}

Reliability requirements:the operation of the medical insur ance service intelligent monitoring system can guarantee $t$ he service of the hospital,the Health Institute and other uni ts for a month without more than two failures.

Availability:helping hospitals and doctors to provide better services for patients, and intelligent monitoring of $t$ he medical treatment activities of doctors and hospitals to enable relevant departments to master the real situation,a void the waste of medical resources and allocate unreaso nable conditions.

\subsection{Error handling requirements}

Set the service area to accept error related notifications, sh ow the user error prompt and give feedback when the erro $r$ is wrong, and provide querying information and code for the error handling personnel.

There will be a problem of error in the distribution of indicators. The backstage will promptly pass the inquiry o $\mathrm{f}$ the prescription list,the prescription summary table and so on,and combine the actual situation of the hospital to $g$ et the distribution of the indexes of the departments and $\mathrm{d}$ octors, and finally correct the data.

Data type error occurs,refers to age more than 100 or less than 0,connection mode format input error,or input $\mathrm{c}$ orrect but not successful completion of the operation, at th is time the judgment is internal or external causes of the $s$ ystem, and finally gives the solution.

\subsection{Interface requirements}

User interface requirements:in addition to the registration information,other related information and treatment infor mation are entered by the doctor to enter the system.After the data enter the system,the administrator carries out the $r$ elated monitoring and processing.

\subsection{Constraints}

The intelligent monitoring system of medical insurance service is based on the Windows operating system, which takes eclipse plus JDK as the development tool,and can be carried out in a single machine or network environment, using the JAVA language.

\subsection{Reverse demand}

The main function of the system is to track and monitor th e medical information of the medical insurance patients,to make statistics,analysis and contrast of the data,and to pre vent the irrational treatment. When the abnormal situation is found,the system will be prompted or warned.

\subsection{Functional requirements}


Combined with the business process of health insurance, $t$ he main functions of the monitoring system are as follows:
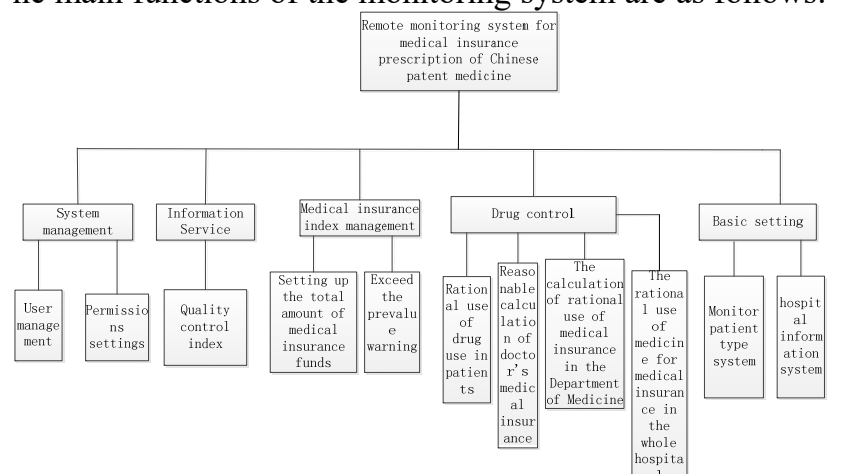

Figure 1. Remote monitoring system for medical insurance prescription of Chinese patent medicine.

(1)System management:This function module mainly includes user management,permission settings and pass word modification.

(2)Information inquiry:Providing user information an $\mathrm{d}$ various quality control indicators for medical insurance management;

(3)Medical insurance index management:The total a mount of medical insurance funds can be set up in the me dical insurance setting, and it can be decomposed to depar tments,groups and doctors by layer by layer,and the amo unt of the amount of medicine for each doctor or patient, $t$ he total amount and the limit of the quantity of drug use a re set;

(4)Drug control:

Data on the following cases are calculated:

The calculation of rational drug use of medical insura nce patients(monthly,quarterly, half a year,one year);

Doctor's reasonable calculation of medical insurance (monthly,quarterly, half a year,one year);

Department of health insurance rational drug use calc ulation(monthly,quarterly, half a year,one year);

The calculation of rational drug use in the whole hosp ital (monthly,quarterly, half a year,one year);

The column layout of each doctor's prescriptions was used to rank doctors to remind doctors to use drugs ration ally.

(5) Basic setup:This functional module needs to set $u$ $p$ the monitoring patient type and export the detailed reco rd of the monitoring object from the hospital information system.

\subsection{Performance requirements}

The system should do the analysis and statistical processin $\mathrm{g}$ of the medication situation immediately after the doctor enters the patient's treatment information.Accordingly, the doctor's medication column chart,the drug list and the tota 1 medical insurance cost statistics should be generated for the administrators to be managed.

\subsection{Possible future demand}

On the basis of the existing basis,we need to expand the fu nction of the system and optimize the system. We can ratio nalize the data by using more efficient algorithms to get $\mathrm{m}$ ore convincing data,and get better service from the medic al insurance object.

\section{System design}

\subsection{Data object}

The object of the system:doctors,patients,hospitals, admin istrators, the use of a single drug, the rational use of medici ne for medical insurance,the excess of the amount of drug $\mathrm{s}$ in a single month or a year, medical insurance index,dru $\mathrm{g}$ ratio (the amount of drug / total amount) and so on.

\subsection{Contact}

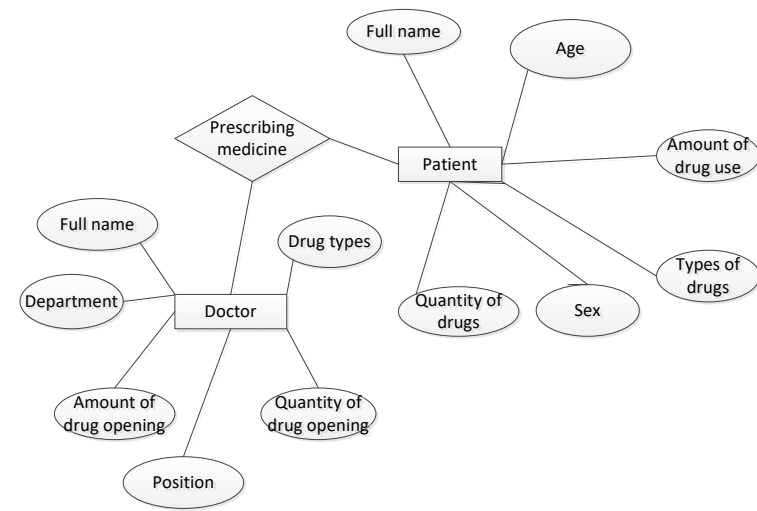

Figure 2.E-R diagram of the relationship between doctors and patients.

\subsection{Main plate design}

The basic functional modules of the medical insurance pre scription monitoring system are mainly composed of the a uxiliary diagnosis module,the basic setting module, the dru $\mathrm{g}$ monitoring module,the prescription management modul e and the query module.

\subsubsection{System management}

1)User management

a.This system mainly includes three types of users: managers, doctors and patients.

b.Managers can manage all user information,includi ng adding,deleting and modifying personnel information, and resetting the staff password.

2)Authority management

a.The system is based on role assignment and divide $\mathrm{d}$ into three roles:manager,doctor and patient according $\mathrm{t}$ o user type.

b.The system administrator has all the permissions o $\mathrm{f}$ the system and can modify other users' privileges.It can play an important role in monitoring and analyzing docto rs' work.

c.Doctors have prescriptions to check their prescripti ons and inquire about the medication of their patients. 


\subsubsection{Auxiliary diagnosi}

For some common symptoms,auxiliary doctor diagnosis,e nter some common symptom data,can help doctors quickl y diagnose.

\subsubsection{Drug control}

1)The list of drugs

According to the actual situation of prescribing the medicine,such as the name,source,prescription,quantity, a mount and time of the drug, the drug is arranged and pres ented.

2)Amount ranking

Drug use ranking.

The number of drugs for a month.

The number of drugs for three months in a row;

The number of drugs for a year in a row.

Through these,we can judge whether there is an over standard situation and achieve a macroscopic monitoring.

3)Proportion calculation

The proportion of the amount of drugs in 2015 is the proportion of all the amounts;

The proportion of the amount of drugs in 2016 is the proportion of all the amounts;

The proportion of the amount of drugs in 2017 is the proportion of all the amounts;

Here, the data in the system database are selected for three years and analyzed.

4)Contrast

Compared with the different years of the single drug, the use of drugs in the same period of time will not be ver $\mathrm{y}$ large, if there is a large fluctuation, it is doubted that ther $\mathrm{e}$ is an irrational behavior of drug opening.

\subsubsection{Prescription management}

The data prescription of proprietary Chinese medicine is managed,and all aspects of data are recorded and recorded in all aspects.

\section{Characteristics and function description of the prescription monitoring system}

(1)The system can greatly improve the work efficiency and the quality of work.The doctor does not have to check the history of medical records repeatedly, and it can ensure the correct and rational use of drugs,and the safety is obviously improved.

(2)Through the system to improve the accuracy of drug delivery to reduce errors and input is not standardized, pharmacy dispensing in the dispensing of mismatching,matching or less distribution,the average monthly number of wrong prescriptions,to bring a great impact on the work.

(3)The system is convenient to inquire, and the electronic prescription of rational drug use can be used to inquire about the patient's previous medication,so that it can avoid repeated use of drugs and effectively promote the rational use of drugs.
(4) Through this system,the quality of medical service has been improved and patient satisfaction has been increased.

\section{Conclusion}

In the stage of system design and implementation,this paper expounds the system architecture and the requirements of the software and hardware environment,gives the function design and the design of the database,and describes the process of the system modeling,design and implementation in detail.After completing the system,the function of the system is tested.The test results show that the system meets the test requirements.

The system has good interface layout and completes functions. However,because of the late start, more content and wide range of medical insurance prescription monitoring system,the research and development of each module need a lot of theoretical knowledge and practical experience, and the direction is a specific drug group.At present,it only realizes some basic functions, some functions may still have some defects, it also needs to be found.It should be constantly tested and improved in the actual application process.

\section{Acknowledgment}

This research was supported by the National Natural Scie nce Foundation of China (No.61762051, No.61363042, N 0.61562045 )、 the Jiangxi province science and technolog y plan projects (No.20151BBE50074, No.20171BBG701 08, No.20171ACE50021) 、 the Project of Jiangxi Health Planning Commission (No.20165537, No.2017A284, No. 2017A313, No.20185516).

\section{References}

1. Felink Peters.A Typology of the Unintended Conseq uences of Drug Crop Reduction[J].Journal of Drug I ssues, 2013,(5):48-50.

2. PM O'Malley,JG Bachman,LD Johnston.Internal-Ext ernal Control and Drug Use among Junior High Sch ool Students in a Rural Community[J].Substance Use \&Misuse, 2013,114-116.

3. Daniel J.Roberts.Drug purchasing by public bidding and internal control management in our hospital[J].M odern medical,2014,(O1):41-42.

4. Landherr A,Friedl B,Heidemann J.A critical review of centrality measures in social networks[J].Business \&Information Systems Engineering,2010,2(6):371-3 85.

5. Kantardzic.Data Mining:concepts,models,methods,a nd algorithms[M].John Wiley \& Sons,2011:56-63.

6. Apache Hadoop[OL].http://hadoop.apache.org/,2014.

7. Kotsiantis SB.Decision trees:a recent overview[J].Ar tificial Intelligence Review.2013,39(4):261-283. 